\title{
Microwave-assisted synthesis and characterization of microstar shaped zinc oxide
}

\author{
Flor Palomara , Miguel José Yacamán e Idalia Gómez * \\ ${ }^{a}$ Universidad Autónoma de Nuevo León, UANL, Fac. de Ciencias Químicas, Lab. De Materiales I, Edif. CELAES 2do. Piso, Av. \\ Universidad s/n Cd. Universitaria, San Nicolás de los Garza, N.L. México. \\ ${ }^{b}$ Department of Physics and Astronomy, University of Texas of San Antonio, Texas, 78249, USA \\ *E-mail: idaliagomezmx@yahoo.com.mx \\ Recibido 23 de abril de 2012, Aceptado 21 de mayo de 2012
}

\begin{abstract}
Resumen
A microwave-assisted solution-phase approach has been applied for the synthesis of zinc oxide microstructures. The synthesis procedure was carried out by using the reagents: Zinc nitrate and Methenamine, at stoichiometric ratio. Analysis by means of X-ray Diffraction (XRD) shows a crystalline phase in hexagonal wurtzite arrangement for $\mathrm{ZnO}$. The presence of microstar shaped zinc oxide $(2-3 \mu \mathrm{m})$ with nanorods $\nmid 50 \mathrm{~nm})$ arranged has been confirmed from High Resolution Scanning Electron Microscopy (HRSEM). The formation of nanorods was confirmed by Transmission Electron Microscopy. In Raman spectroscopy a red shift was detected in the microstructures compared with ZnO bulk. High crystalline materials without additional post-synthesis treatment were found.
\end{abstract}

Palabras clave: $\mathrm{ZnO}$, microstructures, microwave synthesis.

\section{Introduction}

Zinc oxide is a wide band gap $(3.37 \mathrm{eV})$ semiconductor with large binding energy and possesses unique properties. The zinc oxide material has found numerous applications, such as gas sensors [1]; UV photodiodes [2]; piezoelectric devices [3]; varistors [4]; acoustic wave devices [5]; transparent electrodes [6]; facial powders [7]; etc. Semiconductor nanostructured materials have been prepared by serious methods such as microwave heating [8]; nonaqueous approaches [9]; chemical-precipitation [10]; sol-gel process [11]; gas condensation [12]; hydrothermal process [13]; aerosol spray process [14]; and hydrolysis in polyol medium [15]. Microwave synthesis is a synthesis method which entirely differs from the other synthesis techniques. In the microwave technique, a radiation in the wavelength of microwaves penetrates to the material and it is heated volumetrically. This causes the stabilization of the bulk temperature to be reached during microwave processing. It means that the microwave energy penetrates inside the bulk material, which is heated and the heat energy dissipates to the surface of the material. But in case of conventional heating a reverse of heat flow has occurred. Microwave synthesis is promising due to its unique effects such as energy saving, higher reaction rates, rapid volumetric heating, higher selectivity and higher yields of products. Compared to the conventional heating process, (a) microwaves generate higher power densities, enabling increased production speeds and decreased production costs. (b) Microwave energy is precisely controllable and can be turned on and off instantly, eliminating the need for warm-up and cool-down and (c) microwave energy is selectively absorbed by areas of greater moisture resulting in more uniform temperature and moisture profiles, improved yields and enhanced product performance. The interaction between the microwave (MW) radiation and the solution containing the precursor salt, produce a fast heating. Basically, two absorption mechanisms are present: dipole rotation and ion migration. In the first mechanism, the electric dipoles will align with the applied external field and oscillate at the microwave frequency, this produces a damped molecular motion that increases the temperature of the solution; therefore changing the electromagnetic field will produce a permanent rotation of the molecules which finally will increase the temperature of the solution. In the second mechanism, ionic migration is produced by migration of charged particles (ions) in the direction of the electromagnetic field. When the microwave radiation penetrates the solution, their power dissipation is fairly uniform throughout the solvent. Water has a very high dipole moment which makes it one of the best solvents for microwave assisted reactions. The microwave technique has become a powerful tool for production of nanoparticles due to the accelerated dynamics processes present during the synthesis.

\section{Experimental Procedure}

The microwave technique used to obtain microstructures of $\mathrm{ZnO}$ employs a conventional microwave oven with applied power of $1000 \mathrm{~W}$ at a frequency of $2.5 \mathrm{~Hz}$. $\mathrm{Zn}\left(\mathrm{NO}_{3}\right)_{2} \cdot 6 \mathrm{H}_{2} \mathrm{O}$ and $\mathrm{C}_{6} \mathrm{H}_{12} \mathrm{~N}_{4}$ mixture at $1: 1$ concentration in an aqueous solution were used in the processes. Times from 1 to 3 minutes were studied. The samples obtained were washed with deionized water. After, all samples were analyzed by XRD diffraction, RAMAN spectroscopy, HRSEM and TEM. 


\section{Results and Discussion}

The spectrum by XRD of the sample obtained at 1 minute is show in figure 1 . In it can see a perfectly matching with the hexagonal wurtzite structure. The planes (100), (002), (101), (102), (110), (103), (200), (112) and (201) observed in the sample are according with the reported in JCPDS 36-1451 [16]. The samples obtained at 2 and 3 minutes presented similar spectra. The samples were analyzed by HRSEM, figure 2 present four images of the sample obtained at 1 minute. All images have shown the morphology of star-like shaped $\mathrm{ZnO}$ nanostructures. The formation of star morphology consists of sharp rods which look like stars as can see clearly in figure 2C) and 2D). The formation of the star-like shape is probably due to the peculiar property of the methenamine, which acts as a ligand and capping agent. After more, there are some reports that when $\mathrm{C}_{6} \mathrm{H}_{12} \mathrm{~N}_{4}$ was added to the solution with the increase of temperature, $\mathrm{Zn}(\mathrm{OH})_{2}$ precipitated, $\mathrm{ZnO}$ nuclei were formed from the precipitate and then $\mathrm{ZnO}$ nanorods grew [17]. The sizes of the stars are already of $2-3 \mu \mathrm{m}$.

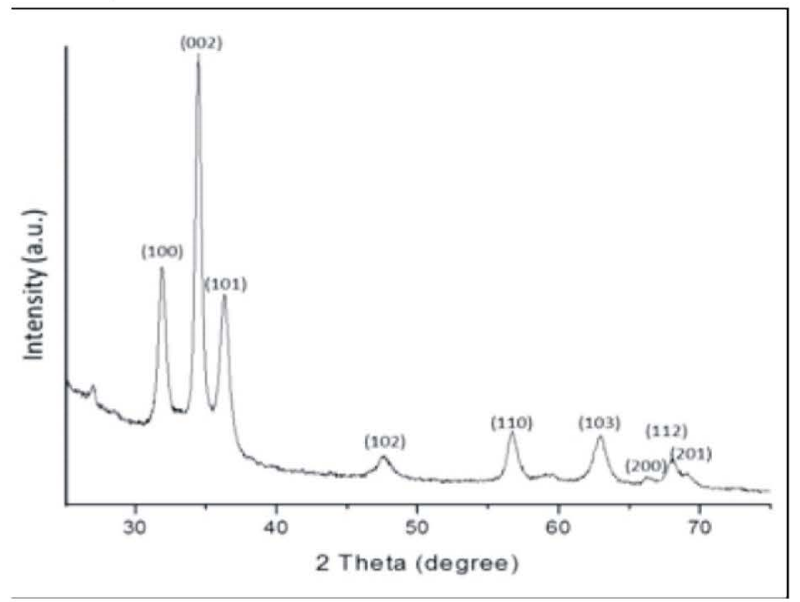

Figure 1. XRD spectrum of zinc oxide sample obtained at 1 minute.

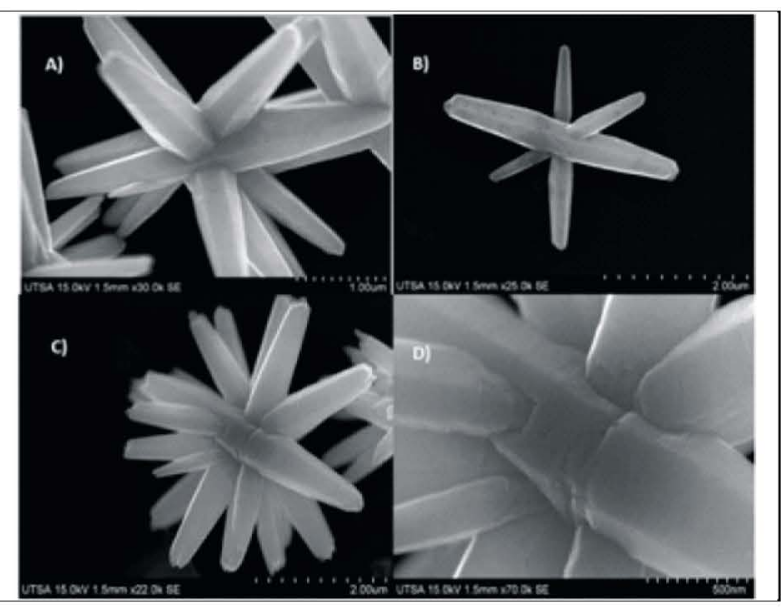

Figure 2. HRSEM images of zinc oxide sample obtained at 1 minute.
In figure 3 is show an image from TEM. In it can see the structural arrangement of one of the nanorods that conforms the star morphology of the $\mathrm{ZnO}$. This has a diameter that starts at $50 \mathrm{~nm}$.

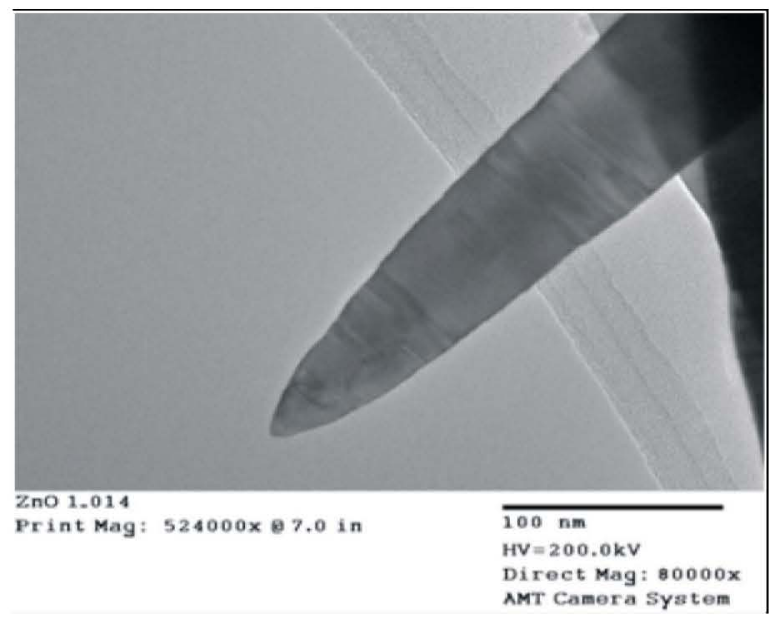

Figure 3. TEM image of zinc oxide sample obtained at 1 minute.

$\mathrm{ZnO}$ is a semiconductor with wurtzite crystal structure. Wurtzite structure belongs to the space group $\mathrm{C}_{6 \mathrm{v}}^{4}$ with two formula units per primitive cell, where all atoms occupy $C_{3 v}$ sites. The Raman active zonecenter optical phonons predicted by the group theory are $A_{1}+2 E_{2}+E_{1}$. The phonons of $A_{1}$ and $E 1$ symmetry are polar phonons and, hence, exhibit different frequencies for the transverse-optical TO and longitudinal-optical LO phonons. Nonpolar phonon modes with symmetry $\mathrm{E}_{2}$ have two frequencies, $\mathrm{E}_{2}$ (high) is associated with oxygen atoms and $E_{2}$ (low) is associated with $\mathrm{Zn}$ sublattice. All described phonon modes have been reported in the Raman-scattering spectra of bulk $\mathrm{ZnO}[18,19]$.

The measured nonresonant Raman spectra of $\mathrm{ZnO}$ microcrystals and bulk samples are shown in Figs. 4a and $4 \mathrm{~b}$, respectively. All spectra in Figure 4 were taken under the laser spot area of $1.6 \mathrm{~m}^{2}$ while the excitation laser power was kept at $15 \mathrm{~mW}$. Comparing the spectra, we can conclude that in the bulk $\mathrm{ZnO}$ spectrum the peak at $379 \mathrm{~cm}^{-1}$ corresponds to $A_{1} T O, 410 \mathrm{~cm}^{-1}$ corresponds to $\mathrm{E}_{1} \mathrm{TO}$, and $439 \mathrm{~cm}^{-1}$ corresponds to $\mathrm{E}_{2}$ high. In the microstars spectrum, the peak at $436 \mathrm{~cm}^{-1}$ corresponds to $\mathrm{E}_{2}$ high, which is shifted by $3 \mathrm{~cm}^{-1}$ compared to bulk. The broad peak at about $330 \mathrm{~cm}^{-1}$ seen in both spectra in Fig. 4 is attributed to the second-order Raman processes. Since the microcrystal size is relatively large, the observed redshift of the $E_{2}$ high phonon cannot be ascribed to the optical-phonon confinement by the nanorods boundaries[20,21]. 


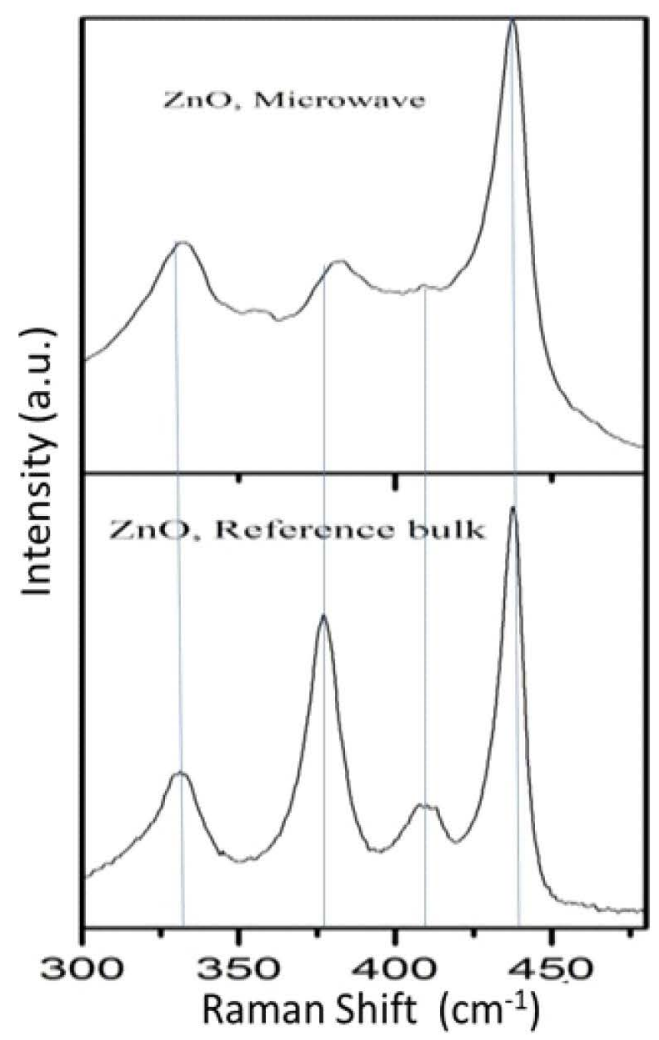

Figure 4. Nonresonant Raman spectra for a) $\mathrm{ZnO}$ nanocrystals; for b) bulk $\mathrm{ZnO}$ a plane. Photoluminescence background is subtracted from the bulk $\mathrm{ZnO}$ spectrum.

\section{Conclusions}

In this work, has been demonstrated that a facile and inexpensive microwave treatment of a zinc nitrate precursor led to the crystalline $\mathrm{ZnO}$ microstars shaped particles when zinc nitrate and methenamine solution was used. One minute was enough in order to obtain the synthesis of $\mathrm{ZnO}$ with wurtzite arrangement.

High crystalline materials were readily obtained without the need of a post-synthesis treatment. This method can also be used for the preparation of other metal oxide powders.

\section{Acknowledgments}

The authors express their gratitude to CONACYT, PAICYT and NSF for the financial support for this work.

\section{References}

1. Bhooloka Rao B. Mater Sci Phys 2000;64:62-5.

2. Lei Luo, Yanfeng Zhang, Mao Samuel S, Liwei Lin. Sens Actuators A2006; 127, 201-6.

3. Gardeniers JGE, Rittersma ZM, Burger GJ. J Appl Phys 1998; 83,7844-54.

4. Dutta S, Jackson HE, Boyd JT, Hickernell FS, Davis RL. Appl Phys Lett 1981, 39206-8.

5. Chang SJ, Su YK, Shei YP. J Vac Sci Technol A 1995, 13 385-8.

6. Horsthuis WHG. Thin Solid Films 1986, 137, 185-92.
7. Liu M, KitaiAH, Mascher P. J Lumin 1992, 54, 35-42.

8. Krishnakumar T, Pinna Nicola, Prasanna Kumari K, Perumal K, Jayaprakash R. Mater Lett 2008, 62, 3437-40.

9. Clavel Guylhaine, Willinger Marc, Zitoun David, Pinna Nicola. Adv Funct Mater 2007, 17, 3159-69.

10. Iwasaki M, Inubushi Y, Ito S. J Mater Sci Lett 1997, 16, 15035.

11. LaufRJ, Bond WD. Am Ceram Soc Bull 1984, 3, 278-81.

12. Eilers H, Tissue BM. Mater Lett 1995, 24, 261-5.

13. Nishizawa H, Yuasa K. J Mater Sci Lett 1998, 7, 985-7.

14. Milosevic O, Uskokovic D. Mater Sci Eng A 1993, 168, 24952.

15. Jezequel D, Guenot J, Jouini N, Fievet F. J Mater Res 1995, 10, 77-83.

16. J. Yang, J. Lang, L. Yang, Y. Zhang, D. Wang, H. Fan, H. Liu, Y. Wang, M. Gao, J. Alloys Compd. 2008, 450, 521-24.

17. Vaezi MR, Sadrnezhaad SK. Mater Des 2007, 28, 515-9.

18. N. Ashkenov et al., J. Appl. Phys. 2003, 93, 126.

19. J. F. Scott, Phys. Rev. B 1970, 2, 1209-211.

20. V. A. Fonoberov and A. A. Balandin, Phys. Rev. B 2004, 70, 195410-16.

21. V. A. Fonoberov and A. A. Balandin, Phys. Status Solidi C $2004,1,2650-53$ 